\title{
Investigation into the Surface Topography of Coated Paper related to Calender
}

\author{
Ying $\mathrm{Li}^{1, \mathrm{a},}$ Qinming Wang ${ }^{1}$, Wenjuan $\mathrm{Gu}^{1}$ and Banggui $\mathrm{He}^{1}$ \\ ${ }^{1}$ Faculty of Mechanical and Electrical Engineering, Kunming \\ University of Science and Technology, Kunming, 650093, China \\ ahaishanying@126.com
}

\begin{abstract}
.
What happens in the surface topography of the paper is of great important for appearance of final printing. The objective of this study was to investigate into the coating surface topography related to calender. The surface chemical property was explored by OCA to obtain the surface free energy. The surface structure was explored in the method of numerical and visual analysis using mercury porosimetry measurement and scanning electron microscopy (SEM). The results indicated that the calender treatment affected the surface chemical characteristic, pore size, pore depth and pore distribution of the coated paper surface. It could be concluded that calender could increase the smoothness, gloss and print density of coated paper, and meanwhile calender could decrease the surface water hydrophilic and increase the surface water repellency with calender pressure increasing.
\end{abstract}

Keywords: Calender; Surface Topography; OCA; Contact angle; Surface free energy; SEM

\section{Introduction}

The substrate properties and ink formulation are key variables to printing quality [1]. The formation of ink film with the desired optical density on paper substrates is consequentially dependent on the ink receptivity by paper, which is determined by structure and optical properties of paper surface [2, 3]. The main objective of this study was to investigate into the effect of calender on surface topography of coating layer. To this aim, the coated samples with different 
calender pressure were studied, by measuring the surface topography of these coatings. To observe surface topography and porosity on the coating surface and obtain the relationship between calender and surface structure, the surface structure was thoroughly analyzed by scanning electron microscopy (SEM) and mercury porosimetry measurment.

\section{Experimental}

Coating and printing. The basis weight of the base paper (supplied by Dong Tang Paper Mill) was $78 \mathrm{~g} / \mathrm{m}^{2}$. The coatings of paper were composed with 100 parts kaolin pigment and different parts carboxylic styrene-butadiene latex (supplied by BASF Company, China) with typical types of appropriate additives. The coating formulations were in Table 1. Coating was performed with a bar coater (model K303 Multi-coater, RK Print Coat Instruments Ltd, United Kingdom). Coating speed was $5 \mathrm{~m} / \mathrm{min}$ and coating thickness was $24 \mu \mathrm{m}$. The coated paper was moved to a drying oven for one minute drying with $140^{\circ} \mathrm{C}$ drying temperature. A laboratory calendar was used to treat the samples with Calender linear pressure of $50 \mathrm{KN} / \mathrm{m}$ and $100 \mathrm{KN} / \mathrm{m}$. The operations were carried out under the following conditions. Ink was offset printing fast-drying cyan ink (TOKA ink, Japan). Ink was transferred onto the coated paper strips using a laboratory printing tester (model IGT Global standard Tester 2, America) and ink distribution apparatus (model IGT Speed Inking Unite 4) with 500N printing pressure and $0.2 \mathrm{~m} / \mathrm{s}$ printing speed. The amount of ink transferred onto the ink distributing roller using IGT ink injector was $0.3 \mathrm{ml}$ and the compensation was $0.075 \mathrm{ml}$ every time.

Measurement of Contact Angle and Surface Free Energy. Contact angle measurements were carried our using apparatus equipped with a video camera and computer software(OCA 20 and SCA 20,Data Physics Instruments). Both coated surface of sampleG1, G2and G3 were investigated. Distilled water was used as probe liquid. Distilled water droplet was deposited on the paper surface by a micro-syringe. Contact angle value was measured after reaching its 
equilibrium on the paper surface. The surface free energy was calculated from contact angle values for coated or non-coated surface of the paper samples.

Table 1 Coating color recipes

\begin{tabular}{ccccc}
\hline Sample & $\begin{array}{c}\text { Kaolin } \\
\left(\mathrm{pph}^{*}\right)\end{array}$ & $\begin{array}{c}\text { SB } \\
\left(\mathrm{pph}^{*}\right)\end{array}$ & $\begin{array}{c}\text { Solids } \\
(\%)\end{array}$ & $\begin{array}{c}\text { Calender } \\
(\mathrm{KN} / \mathrm{m})\end{array}$ \\
\hline A & 100 & 15 & 60 & 0 \\
B & 100 & 15 & 60 & 50 \\
C & 100 & 15 & 60 & 100 \\
\hline
\end{tabular}

SEM. Scanning electron microscopy (Hitachi S3700N, Japan) was utilized to characterize the surface topography of the coating layer. For SEM observation, all coating samples were gold coated and $10 \mathrm{KV}$ beam energy was used to obtain surface topography images in order to reduce the possibility of any thermal damage.

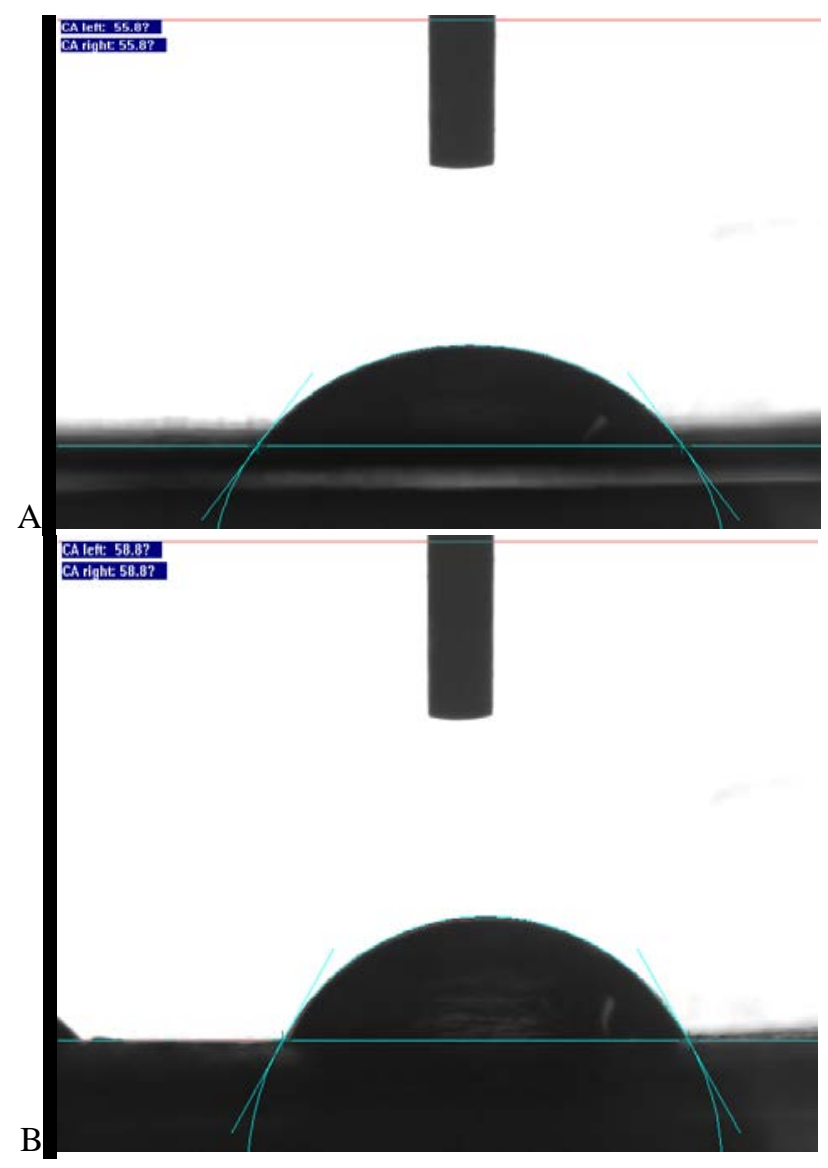




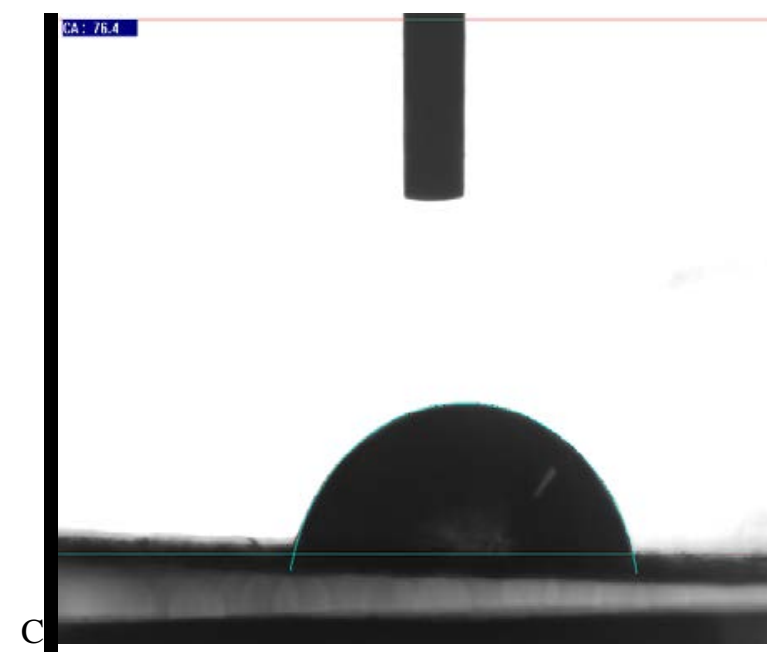

Fig.1 Contact angle measured by OCA

Mercury Porosimetry. Coating pore size distributions were determined by mercury porosimetry Measurment which was a Quantachrome Poremaster.

\section{Results and Discussion}

Chemical property of Coated Paper. To characterize the chemical property of different coating surface, contact angle measurements were used. The contact angle could be described by the Young equation [4]. The balance of the forces (surface tensions) acting the triple line Young's equation [5, 6]. Fig.1 showed images of interactions of liquid and solid in this experiment for the different coating surface. The contact angles and surface free energy of all the coatings were quite different shown in Fig.2. The contact angle values of sample A, B and $C$ with different coating surface were $55.5^{\circ}, 58.8^{\circ}$ and $76.4^{\circ}$. Surface free energy could show the surface chemical properties. Surface free energy was high and surface water hydrophilic was good, but Surface free energy was low and surface water repellency was good. The results indicated the surface free energy of sample C was lowest and surface water repellency was highest. Surface free energy of sample A was highest and surface water hydrophilic was highest. It could be concluded that contact angle increased with calender pressure increasing, which indicated that surface water hydrophilic decreased and 
repellency increased with calender pressure adding.

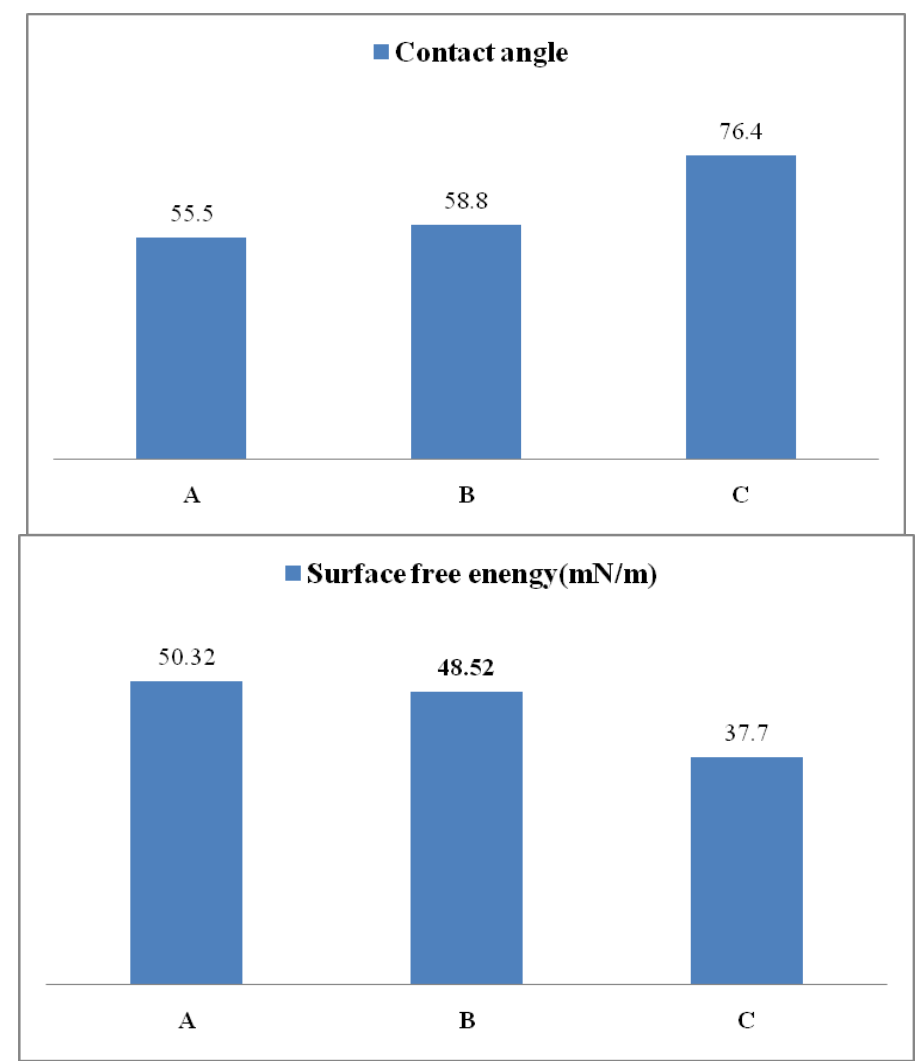

Fig.2 Contact angle and surface free energy of coated samples

Surface Topography of coating layer. The surface topography of samples were examined by SEM. Changes in the surface topography of samples with the different calender condition were shown in Fig.2. It could be seen from Fig.2 that these samples differed largely in surface topography maps. For all coating samples investigated, with no calender pressure of sample A the kaolin pigment particles were relatively apart and arranged randomly. With calender pressure increasing, the arrangement of the kaolin pigment particles got closer together and there were more and bigger flat clay plates which could be observed. The surface of samples $C$ was smoother and had fewer pores than the other surfaces. 


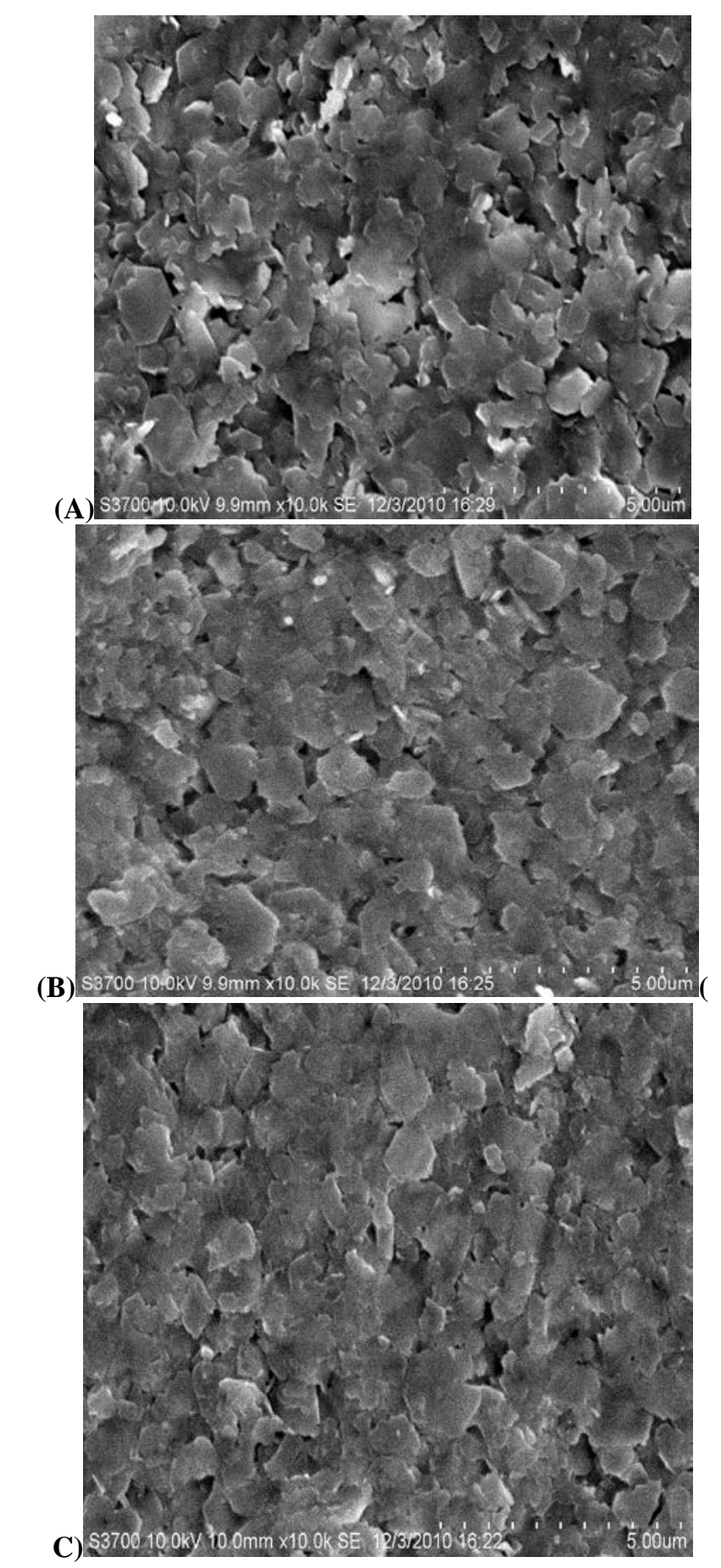

Fig.2. Typical SEM images of surface topography of. (Magnification: 10000) 
Table 2 Pore size distribution of coated paper

\begin{tabular}{cccc}
\hline Sample & A & B & C \\
\hline Volume intruded $(\mathrm{ml} / \mathrm{g})$ & 0.4517 & 0.3712 & 0.3219 \\
Median pore diameter $(\mu \mathrm{m})$ & 1.9193 & 1.7782 & 1.7105 \\
Mean pore diameter $(\mu \mathrm{m})$ & 0.7632 & 0.6020 & 0.5532 \\
Porosity $(\%)$ & 38.7639 & 30.0435 & 25.1585 \\
Air permeability $(\mu \mathrm{m} / \mathrm{Pa} . \mathrm{s})$ & 0.6102 & 0.5085 & 0.4294 \\
\hline
\end{tabular}

Relationship between Surface Microstructure and Calender. The surface pore distributions of samples were examined by mercury porosimetry measurment. It suggested form Table 2 that these samples differed largely in pore size distribution. Sample C had the lowest porosity, mean pore diameter, volume intruded and air permeability, which suggested that $C$ was smoother than sample A and B, which showed the results were in accord with the results tested by SEM. Median pore diameter of sample A was the largest, which indicated that sample A had more small pores than the other samples. The conclusions were obtained that distribution and size of pore were different due to the different calender pressure. It could be concluded that the coating surfaces became smoother with the calender pressure increasing.

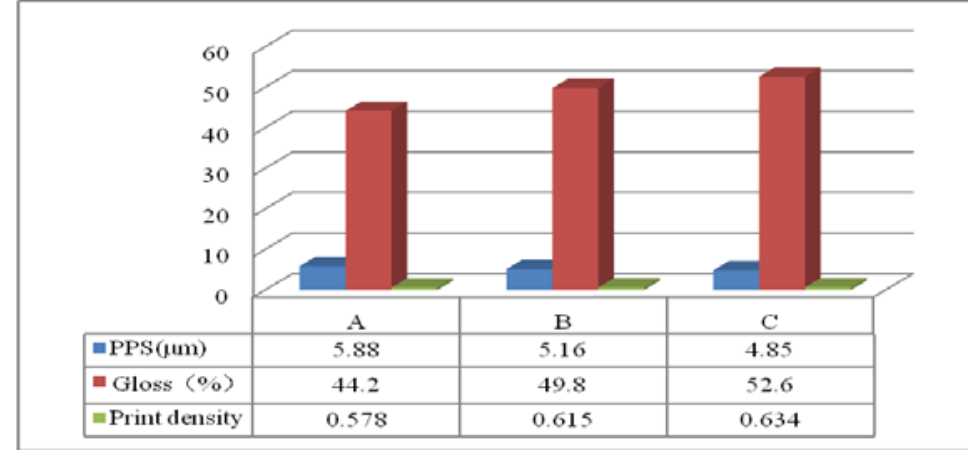

Fig.3. Print density of samples

Effect of Calender on Print Density of Coated Paper. Fig.3 summarized the evaluated mean values (calculated from four measurements) of surface properties for coated paper samples used in the study. The results obtained were explored as the basis for discussion about the behavior of ink transferred onto the coated paper substrates. It could be seen from Fig.3 that the roughness was declined through calendaring treatment. Calendering could obviously improve the smoothness and gloss of coated paper. The print density of samples printed on the same condition was indicated in Fig.3. Sample C had the highest print density. In contrast, Sample A had the lowest print density. It suggested that print density increased with the adding of calendar pressure. It could be concluded that calender caused higher print density. In despite of lower ink absorption of calendered paper was yielded, higher print density occurred comparing with uncalendered coated paper. It was due to surface treatment that calender caused closed porosity which prevented the penetration of ink. The lower amount of ink penetrated into paper coating and higher amount of ink set on the surface of coated paper, which resulted in that the lower amount of ink produced higher 
print density. The smoother and higher gloss surface of calendered paper could improve the printing quality and decreased the amount of ink usage.

\section{Conclusions}

The structure of the coating layer has a strong influence on the optical and physical properties of paper as well as on the print quality. Investigation into the relationship between surface topography and calender was obtained in this research. The findings were obtained that calender affected surface chemical property, pore size, depth and distribution of the coated paper surface owing to the pigment accumulating condition on the coating surface with the different calender pressure. The conclusions were drawn that calender treatment could contribute to improve the coating surface topography and the formation of even pore size and distribution, which was good to the paper surface characteristics and resulted in low roughness, high gloss and high print density. Surface water hydrophilic decreased and repellency increased with calender pressure adding.

\section{Acknowledgements}

The authors express their gratitude to the Yunnan Province Science and Technology Department (Project KKSY 2012/01059 and KKSY 2012/01051) for financial support.

\section{References}

[1]G. Engstrom, M. Rigdahl: Nord. Pulp Pap. Res. J, 1992, 7(2) (1992), p.55.

[2] R. Allem: J. Pulp Pap. Sci. 24 (10)(1998), p.329.

[3]Y. H. Zang, S. AsplerJ: J. Pulp Pap. Sci. 24 (5)(1998), p.141.

[4] G. Whyman, E. Bormashenko, T. SteinElton: Chem. Phys. Lett. 450 (2008), p.355.

[5] B. He, J. Lee, N. A.Patankar: Collids Surf. A. 248(2004) p.101.

[6] P. Letellier, A. Mayaffre, M. Turmine: Colloid Interface. Sci. J. 314(2007), p.604. 